\title{
Perfil de pacientes internados em uso de sonda nasoenteral e fatores associados ao óbito
}

\author{
Profile of patients admitted using a nasoenteral tube and factors associated with death
}

\section{DOI: $10.37111 /$ braspenj.2020353013}

Fernanda Raphael Escobar Gimenes de Sousa' Flávia Helena Pereira ${ }^{2}$

Julia Maria Aguiar ${ }^{3}$

Paulo Janazi ${ }^{4}$

Patrícia Rezende do Prado

Adriane de Medeiros ${ }^{6}$

\section{Unitermos:}

Intubação gastrointestinal. Nutrição enteral. Morte.

\section{Keywords:}

Intubation, gastrointestinal. Enteral nutrition. Death.

\section{Endereço para correspondência:}

Fernanda Raphael Escobar Gimenes de Sousa Universidade de São Paulo, Escola de Enfermagem de Ribeirão Preto, Departamento de Enfermagem Geral e Especializada.

Avenida Bandeirantes, 3900 - Ribeirão Preto, SP, Brasil - CEP 14040-902

E-mail: fregimenes@eerp.usp.br

\section{Submissão}

2 de junho de 2020

Aceito para publicação

4 de setembro de 2020

\section{RESUMO}

Objetivo: Analisar o perfil de pacientes em uso de sonda nasoenteral (SNE) e os fatores associados ao óbito. Método: Estudo transversal e analítico, realizado em clínica médica de sete hospitais brasileiros da região Norte, Nordeste, Sudeste e Sul. A amostra foi constituída por 447 pacientes internados em uso de SNE. Os critérios de inclusão foram: pacientes com mais de 18 anos; com SNE posicionada em região gástrica ou entérica; internados há pelo menos 24 horas. Os dados demográficos, clínicos e terapêuticos foram coletados no período de outubro de 2017 a abril de 2019, obtidos dos prontuários médicos, por meio de um dispositivo móvel (celular ou tablet) e registrados na plataforma Survey Monkey ${ }^{\circledR}$. A complexidade assistencial foi verificada por meio de sistema de classificação de pacientes e a gravidade, por meio do Índice de Comorbidade de Charlson ajustado por idade. Para análise dos dados, foi realizada estatística descritiva, testes Qui-Quadrado de Cochran-Armitage e Exato de Fisher. Resultados: A maioria dos pacientes era homem $(52,2 \%)$, branca $(67,6 \%)$, casada $(45,1 \%)$, com ensino fundamental incompleto $(38,4 \%)$. Em relação ao diagnóstico médico principal, a maioria apresentou doença do aparelho circulatório $(30 \%)$; mais de $50 \%$ estavam em estado de alerta, $44 \%$ eram altamente dependentes dos cuidados de enfermagem e $47,9 \%$ apresentavam alto risco de óbito. Verificou-se significância estatística entre óbito e as variáveis complexidade assistencial, nível de consciência e idade. Conclusões: O conhecimento sobre o perfil dos pacientes em uso de SNE e dos fatores associados ao óbito são importantes, pois oferece dados que permitem melhor planejamento da assistência à saúde.

\section{ABSTRACT}

Objective: To analyze the profile of patients using nasoenteral tube (NET) and the factors associated with death. Methods: Cross-sectional and analytical study was performed in medical wards of seven Brazilian hospitals in the North, Northeast, Southeast and South. The sample consisted of 447 patients hospitalized with NET. The inclusion criteria were: patients older than 18 years; with NET positioned in the stomach or intestine; hospitalized for at least 24 hours. Data were collected from October 2017 to April 2019, using a mobile device (cell phone or tablet). Demographic, clinical and therapeutic data were obtained from medical records and recorded in an electronic form, using the Survey Monkey ${ }^{\circledR}$ platform. The complexity of care was verified through a patient classification system and the severity of the disease, by the Charlson Comorbidity Index adjusted by age. For data analysis, descriptive statistics, Chi-Square Cochran-Armitage and Fisher's Exact tests were performed. Results: Most patients were male (52.2\%), white (67.6\%), married (45.1\%), with incomplete elementary school (38.4\%). Regarding the main medical diagnosis, most of them presented circulatory system disease (30\%); more than $50 \%$ were in a state of alert, $44 \%$ were highly dependent on nursing care and had high risk for death (47.9\%). There was statistical significance among death and the variables care complexity, level of awareness and age. Conclusions: Knowledge about the profile of patients using NET and the factors associated with death are important because it offers data that allow better planning of health care.

1. Professor Associado, Universidade de São Paulo, Escola de Enfermagem de Ribeirão Preto, Departamento de Enfermagem Geral e Especializada, Ribeirão Preto, SP, Brasil.

2. Professora Doutora em Ciências da Saúde, Instituto de Educação, Ciência e Tecnologia do Sul de Minas Gerais (IFSULDEMINAS), Campus Passos, Passos, MG, Brasil.

3. Discente no curso Bacharelado em Enfermagem, Universidade de São Paulo, Escola de Enfermagem de Ribeirão Preto, Ribeirão Preto, SP, Brasil. 4. Discente no curso Bacharelado em Enfermagem, Centro Universitário Estácio de Sá, Ribeirão Preto, SP, Brasil.

5. Professor Adjunto, Universidade Federal do Acre, Departamento de Enfermagem, Rio Branco, AC, Brasil.

6. Doutoranda, Universidade de São Paulo, Escola de Enfermagem de Ribeirão Preto, Programa de Pós-Graduação Enfermagem Fundamental, Ribeirão Preto, SP, Brasil. 


\section{INTRODUÇÃO}

As sondas nasoenterais (SNE) são utilizadas de acordo com critérios específicos, tais como risco ou tratamento da desnutrição e disfunção parcial ou total do trato digestório. Seu uso pode reduzir complicações infecciosas, problemas associados à cicatrização, tempo de internação, custos hospitalares e mortalidade'. Na China, aproximadamente 6,6 milhões de pacientes são submetidos a esse procedimento a cada ano². Já no Reino Unido, segundo a Agência Nacional de Segurança do Paciente, os números são de cerca de 170 mil SNE por ano ${ }^{3}$. No Brasil, esses dados não estão disponíveis, porém sabe-se que esses dispositivos são frequentemente utilizados tanto no contexto hospitalar quanto domiciliar, especialmente em pacientes oncológicos $^{4}$, de terapia intensiva ${ }^{5}$ e internados em enfermarias de clínica médica ${ }^{6}$. Estudo em uma unidade de terapia intensiva brasileira ${ }^{7}$ demonstrou que $40 \%$ dos 907 idosos internados receberam nutrição enteral (NE) via SNE.

Dado que o número de pacientes com condições crônicas aumentou significativamente em todo o mundo 8 , incluindo o Brasil9, e que esses pacientes geralmente necessitam de SNE, é fundamental que os profissionais dos serviços de saúde apropriem-se do conhecimento disponibilizado por evidências para fornecer segurança e cuidado de alta qualidade aos pacientes que necessitam desses dispositivos ${ }^{10}$. Portanto, o conhecimento sobre o perfil dos pacientes em uso de SNE é importante, uma vez que a partir dessa análise promove-se melhor sistematização da assistência de enfermagem. A importância desse conhecimento está relacionada ao direcionamento da assistência prestada a esse tipo de paciente, com especial atenção aos fatores de risco associados, aos eventos adversos e ao prognóstico'.

Há escassez de estudos no Brasil sobre o perfil de pacientes em unidades hospitalares em uso de SNE. Os poucos estudos existentes envolveram análise de dados em uma única instituição ${ }^{1,11}$. Dessa forma, justifica-se a necessidade do desenvolvimento de estudos em nível nacional para melhor compreensão do perfil de pacientes em uso de SNE.

Logo, o objetivo primário foi descrever o perfil demográfico, clínico e terapêutico dos pacientes em uso de SNE. O objetivo secundário foi identificar associações entre óbito e as variáveis independentes: complexidade assistencial, gravidade, nível de consciência e uso de dispositivo invasivo para respiração.

\section{MÉTODO}

\section{Desenho}

Estudo transversal e analítico, proveniente de estudo multicêntrico ${ }^{10}$.

\section{Local de Estudo}

Foi realizado em enfermarias da clínica médica de sete hospitais brasileiros das regiões Norte, Nordeste, Sudeste e Sul. Os hospitais participantes foram: Hospital das Clínicas do Acre (HCA), Hospital Geral de Fortaleza (HGF), Hospital das Clínicas da Faculdade de Medicina de Ribeirão Preto da Universidade de São Paulo (HCFMRP-USP), Hospital Estadual Américo Brasiliense (HEAB), Hospital Estadual Sumaré (HES), Hospital São Vicente de Paulo (HSVP) e Hospital Santa Cruz do Rio Grande do Sul (HSCRGS).

\section{Participantes}

O universo do estudo foi constituído por pacientes internados nas enfermarias de clínica médica dos sete hospitais, que necessitaram de SNE durante a internação ou que foram admitidos nas enfermarias com a sonda. Os critérios de inclusão foram: pacientes com mais de 18 anos e internados há pelo menos 24 horas. Foram excluídos do estudo pacientes que necessitaram de SNE para drenagem e que foram readmitidos nas unidades de internação.

\section{Tamanho da Amostra}

A determinação do tamanho amostral se deu por meio de amostragem aleatória estratificada com alocação proporcional por estratos, em que cada estrato foi formado pelos andares/alas de cada hospital. Adotando-se os parâmetros de erro relativo de 20\%, nível de significância de $5 \%$ e a população total de 6.564 pacientes com SNE, foi calculado um tamanho amostral total de 391 pacientes. Contudo, as observações superaram o tamanho da amostra requerido, totalizando 447 pacientes.

\section{Instrumentos e Variáveis}

instrumento de coleta de dados foi composto por um formulário eletrônico desenvolvido pela equipe de pesquisa $^{10}$. Foi utilizado o servidor Survey Monkey ${ }^{\circledR}$ para a coleta e o gerenciamento de dados (disponível em: https:// pt.surveymonkey.com/r/F67KP5G). As variáveis consideradas no presente estudo foram:

- Demográficas: sexo; cor da pele; estado civil; escolaridade;

- Clínicas: diagnóstico médico principal, segundo o Código Internacional de Doenças (CID-10); gravidade das comorbidades, avaliada pelo Índice de Comorbidade de Charlson (ICC) ${ }^{12}$; complexidade assistencial, avaliada por meio do escore final do Sistema de Classificação de Pacientes (SCP) ${ }^{13}$; nível de consciência, avaliado por meio da Escala ACDU ${ }^{14}$ e existência de dispositivo invasivo para respiração;

- Terapêuticas: dados relacionados à SNE (motivo para o uso, técnica para introdução e técnica para confirmação do posicionamento da sonda); 
- Data e hora da alta; motivo da alta (óbito ou não óbito).

O nível de consciência, nesse estudo, foi verificado por meio da Escala ACDU, que significa: alerta, confuso, sonolento e inconsciente ${ }^{14}$. É uma escala simples, rápida e útil para avaliar, à beira leito, o nível de consciência do paciente.

A complexidade assistencial foi avaliada por enfermeiro treinado, membro da equipe de pesquisa. Foi utilizado o $\mathrm{SCP}^{13}$, que é recomendado pelo Conselho Federal de Enfermagem do Brasil e empregado na maioria dos hospitais participantes do estudo. $O$ instrumento foi desenvolvido com a finalidade de classificar os pacientes segundo o grau de dependência da equipe de enfermagem.

A gravidade dos pacientes foi avaliada por meio do ${ } \mathrm{CC}^{12}$. O estudo original foi realizado a partir de 20 condições clínicas selecionadas empiricamente com base no efeito dessas condições sobre o prognóstico de pacientes internados ${ }^{2}$. Originalmente, o ICC foi proposto para ser utilizado em estudos longitudinais de doenças crônicas, todavia há evidências favoráveis de sua validade para mensurar a gravidade dos pacientes em uma única hospitalização ${ }^{15}$.

Destaca-se que, neste estudo, o ICC foi ajustado em função da idade do paciente (ICC-I), de modo que, a partir dos 50 anos, foi adicionado um peso a cada década de vida ao escore final' ${ }^{12}$. Com base na pontuação final do ICC-I, os pacientes foram estratificados em três grupos: risco leve (pontuação de 1 a 2); risco moderado (pontuação de 3 a 4) e alto risco (pontuação $\geq 5$ ). Esta variável foi obtida do prontuário médico do paciente.

\section{Procedimentos de Coleta de Dados}

Os dados foram coletados no período de outubro de 2017 a abril de 2019. Em cada centro participante do estudo, foi designada uma coordenadora regional com a finalidade de garantir a qualidade e completude dos dados coletados. As coordenadoras também foram responsáveis por recrutar e treinar os assistentes de pesquisa que participaram da coleta de dados. Cada assistente foi submetido a treinamento teórico-prático, com carga horária total de 16 horas (teórico $=4$ horas; prático $=12$ horas). Neste treinamento, foram apresentados os objetivos do estudo, a metodologia adotada, o formulário eletrônico, o guia do observador e as técnicas de coleta de dados.

Foi desenvolvido guia do observador contendo informações gerais sobre o estudo; instruções sobre o acesso ao formulário eletrônico; definição de cada variável e informações adicionais sobre a coleta das variáveis. O guia foi disponibilizado na versão impressa e via e-mail, cujo endereço eletrônico foi criado para cada centro participante do estudo. Assim, todos os integrantes da pesquisa tiveram acesso ao documento. $\bigcirc$ objetivo do guia foi padronizar a coleta de dados.

Os dados foram coletados por meio de um dispositivo móvel (celular ou tablet). Para a análise do perfil demográfico, clínico e terapêutico dos pacientes em uso de SNE, foram obtidos dados da admissão do paciente na enfermaria de clínica médica e registrados no formulário eletrônico de coleta de dados. Dados relacionados à alta do paciente também foram obtidos dos prontuários médicos. Considerando que quatro hospitais participantes do estudo apresentavam prontuário eletrônico, este foi acessado nos computadores disponíveis nas unidades/enfermarias. A consulta aos prontuários médicos ocorreu nos períodos da tarde ou da noite, porque eram os períodos com menor circulação de pessoal.

\section{Análise dos Dados}

Os dados foram transferidos do servidor Survey Monkey ${ }^{\circledR}$ para planilhas do Programa Microsoft Excel ${ }^{\circledR}$. $\mathrm{Na}$ análise estatística descritiva, foram aplicados cálculo de proporções e medidas de tendência central e de variabilidade.

Para verificar a presença de associações entre as variáveis "complexidade assistencial", segundo o SCP; "gravidade", medida pelo ICC-I e "nível de consciência", avaliada pela Escala ACDU, com o óbito, foi utilizado o teste Qui-Quadrado de Cochran-Armitage. Este teste é indicado para as situações quando se têm variáveis ordinais e nominal. $\bigcirc$ teste consiste em detectar uma tendência linear na proporção de "sucessos", ou seja, a proporção de óbitos. No caso do cruzamento entre as variáveis "uso de dispositivo invasivo" (sim/não) no momento do evento "óbito" (sim/não), foi utilizado o teste Exato de Fisher. $\mathrm{Na}$ associação entre "idade", "tempo de sonda", "tempo de internação" e "número de comorbidades", também foi utilizado o teste Qui-Quadrado de Cochran-Armitage. Todas as análises foram realizadas por meio do programa $R$ versão 3.5.3, considerando-se um nível de significância de $5 \%(\alpha=0,05)$.

\section{Questões Éticas}

O estudo multicêntrico foi aprovado pelo Comitê de Ética em Pesquisa da Escola de Enfermagem de Ribeirão Preto da Universidade de São Paulo (EERP-USP) (CAAE: $56166016.3 .1001 .5393)$. Foi entregue aos participantes do estudo o termo de consentimento livre e esclarecido (TCLE) e esses foram informados que os resultados da pesquisa serão destinados às possíveis publicações e que serão garantidos o sigilo e o anonimato. 


\section{RESULTADOS}

Dos 447 pacientes incluídos na pesquisa, a maioria foi constituída por homens ( $n=233 ; 52,2 \%)$, brancos ( $n=292$; $67,6 \%)$, casados ( $n=194 ; 45,1 \%)$, com ensino fundamental incompleto $(n=164 ; 38,4 \%$ ) (Tabela 1). A média de idade foi de 64,91 anos $(66,49 \pm 16,49$ anos) e do tempo de internação foi de 17,32 dias (1 1,25 $\pm 28,98$ dias).

A maior parte dos pacientes ( $n=245 ; 54,8 \%$ ) foi admitida nas enfermarias de clínica médica no período da tarde e com a SNE $(n=285 ; 63,8 \%)$. As comorbidades mais frequentes foram: doença vascular periférica $(n=152 ; 34 \%)$, doença cerebrovascular ( $n=83 ; 18,6 \%)$, diabetes sem complicação $(n=83 ; 18,6 \%)$ e doença renal grave ou moderada $(n=53 ; 11,9 \%)$.

Em relação ao diagnóstico médico principal, a maioria foi admitida com doença do aparelho circulatório $(n=128$; $30 \%)$, seguida por neoplasias $(n=61 ; 14,3 \%)$ e doenças do aparelho respiratório $(n=53 ; 12,4 \%)$. Mais de $50 \%$ (n $=255$ ) dos pacientes encontravam-se em estado de alerta, $44 \%$ ( $n=188$ ) eram altamente dependentes dos cuidados de enfermagem e $47,9 \%(n=194)$ apresentavam alto risco de óbito (Tabela 2).

Tabela 1 - Distribuição das variáveis demográficas dos pacientes em uso de SNE, internados na clínica médica (N=447). Ribeirão Preto, 2019.

\begin{tabular}{|c|c|c|}
\hline \multirow[b]{2}{*}{ Variáveis demográficas } & \multicolumn{2}{|c|}{ Frequência $^{a}$} \\
\hline & $\mathrm{n}$ & $\%$ \\
\hline \multicolumn{3}{|l|}{ Sexo } \\
\hline Feminino & 213 & 47,8 \\
\hline Masculino & 233 & 52,2 \\
\hline \multicolumn{3}{|l|}{ Cor da pele } \\
\hline Amarela (oriental) & 2 & 0,5 \\
\hline Negra & 66 & 15,3 \\
\hline Parda & 72 & 16,7 \\
\hline Branca & 292 & 67,6 \\
\hline \multicolumn{3}{|l|}{ Estado civil } \\
\hline Companheiro & 13 & 3,0 \\
\hline Separado/divorciado & 40 & 9,3 \\
\hline Solteiro & 85 & 19,8 \\
\hline Viúvo & 98 & 22,8 \\
\hline Casado & 194 & 45,1 \\
\hline \multicolumn{3}{|l|}{ Escolaridade } \\
\hline Pós-graduação & 1 & 0,2 \\
\hline Nível superior incompleto & 13 & 3,0 \\
\hline Nível superior completo & 20 & 4,7 \\
\hline Ensino médio incompleto & 22 & 5,1 \\
\hline Ensino médio completo & 35 & 8,3 \\
\hline Não sabe ler e escrever & 42 & 9,8 \\
\hline Ensino fundamental completo & 65 & 15,2 \\
\hline $\begin{array}{l}\text { Não tem escolaridade, mas sabe } \\
\text { ler e escrever }\end{array}$ & 65 & 15,2 \\
\hline Ensino fundamental incompleto & 164 & 38,4 \\
\hline
\end{tabular}

Tabela 2 - Distribuição das variáveis clínicas dos pacientes em uso de SNE admitidos na clínica médica (N=447). Ribeirão Preto, 2019.

\begin{tabular}{|c|c|c|}
\hline \multirow[b]{2}{*}{ Variáveis clínicas } & \multicolumn{2}{|c|}{ Frequênciaa } \\
\hline & $\mathbf{n}$ & $\%$ \\
\hline \multicolumn{3}{|l|}{ CID-10b Principal } \\
\hline $\begin{array}{l}\text { Transtornos mentais e comportamentais } \\
\text { (F00 - F99) }\end{array}$ & 12 & 2,8 \\
\hline $\begin{array}{l}\text { Doenças do sangue e dos órgãos } \\
\text { hematopoiéticos e alguns transtornos } \\
\text { imunitários (D50 - D89) }\end{array}$ & 12 & 2,8 \\
\hline $\begin{array}{l}\text { Doenças endócrinas, nutricionais e } \\
\text { metabólicas (E00 - E90) }\end{array}$ & 13 & 3,0 \\
\hline $\begin{array}{l}\text { Algumas doenças infecciosas e } \\
\text { parasitárias (A00 - B99) }\end{array}$ & 17 & 4,0 \\
\hline $\begin{array}{l}\text { Doenças do aparelho geniturinário } \\
\text { (N00 - N99) }\end{array}$ & 23 & 5,5 \\
\hline Outras ${ }^{c}$ & 23 & 5,5 \\
\hline Doenças do sistema nervoso (G00 - G99) & 25 & 5,9 \\
\hline Doenças do aparelho digestivo (K00-K93) & 29 & 6,8 \\
\hline $\begin{array}{l}\text { Sintomas, sinais e achados anormais } \\
\text { de exames clínicos e de laboratório, não } \\
\text { classificados em outra parte (R00 - R99) }\end{array}$ & 30 & 7,0 \\
\hline $\begin{array}{l}\text { Doenças do aparelho respiratório } \\
\text { (J00 - J99) }\end{array}$ & 53 & 12,4 \\
\hline Neoplasias [tumores] (C00 - D48) & 61 & 14,3 \\
\hline Doenças do aparelho circulatório (100 - 199) & 128 & 30,0 \\
\hline \multicolumn{3}{|l|}{ Nível de consciência (Escala ACDU) } \\
\hline Inconsciente & 34 & 7,7 \\
\hline Sonolento & 46 & 10,4 \\
\hline Confuso & 108 & 24,4 \\
\hline Alerta & 255 & 57,6 \\
\hline \multicolumn{3}{|l|}{ Complexidade assistencial (SCP) } \\
\hline Cuidado intensivo & 17 & 4,0 \\
\hline Cuidado mínimo & 58 & 13,6 \\
\hline Cuidado semi-intensivo & 70 & 16,4 \\
\hline Cuidado intermediário & 94 & 22,0 \\
\hline Alta dependência & 188 & 44,0 \\
\hline \multicolumn{3}{|l|}{ Gravidade (ICC-I) } \\
\hline Risco nulo & 30 & 7,4 \\
\hline Risco leve & 75 & 18,5 \\
\hline Risco moderado & 106 & 26,2 \\
\hline Risco alto & 194 & 47,9 \\
\hline
\end{tabular}

${ }^{a} \mathrm{~A}$ frequência absoluta não totaliza 447 pacientes devido ao número de dados faltantes. ${ }^{\text {b}} \mathrm{Clas}$ sificação Internacional de Doenças, $10^{a}$ edição. "A categoria "Outros" incluiu: Causas externas de morbidade e de mortalidade (V01-Y98), doenças da pele e do tecido subcutâneo (L00-L99), doenças do sistema osteomuscular e do tecido conjuntivo (M00-M99), fatores que influenciam o estado de saúde e o contato com os serviços e o contato com os serviços de saúde (Z00-Z99). 
O principal motivo para o uso da sonda foi a queda do sensório ( $n=157 ; 35,2 \%)$; o método mais utilizado para a introdução da SNE foi a inserção às cegas, à beira leito ( $n=224 ; 50,9 \%$ ) e o método mais empregado para confirmar o posicionamento da extremidade distal da sonda foi a ausculta epigástrica ( $\mathrm{n}=69 ; 18,3 \%$ ) (Tabela 3).

Ocorreu maior número de óbitos entre os pacientes classificados como alta dependência pelo SCP $(n=39 ; 8,7 \%)$ e em risco alto pelo ICC-I ( $n=53 ; 11,9 \%)$. Dentre os pacientes que foram a óbito, apenas sete $(1,6 \%)$ necessitavam de cuidado mínimo de enfermagem e cinco $(1,1 \%)$ apresentaram ausência de risco para o óbito. Com relação ao nível de consciência, 54 (12\%) pacientes estavam com alteração, ou seja, confusos, sonolentos ou inconscientes. Ademais, $18,6 \%(n=83)$ dos pacientes não apresentavam dispositivo invasivo para respiração. Houve associação entre óbito e complexidade assistencial $(p=0,003)$ e nível de consciência $(p=0,009)$ (Tabela 4).

Foram identificados $100(22,4 \%)$ óbitos no período do estudo; a frequência foi maior nas enfermarias de clínica geral $(n=39 ; 39 \%)$, oncologia $(n=21 ; 21 \%)$ e geriatria $(n=11$; $11 \%)$. A média de idade dos pacientes que morreram foi de 68,97 anos (70,24 $\pm 14,63$ anos), do tempo de uso de sonda foi de 14,16 dias $(9,00 \pm 16,04$ dias $)$ e do tempo
Tabela 3 - Distribuição das variáveis terapêuticas dos pacientes em uso de SNE, internados na clínica médica (N=447). Ribeirão Preto, 2019.

\begin{tabular}{|c|c|c|}
\hline & \multicolumn{2}{|c|}{ Frequência } \\
\hline Variáveis terapêuticas & $\mathbf{n}$ & $\%$ \\
\hline \multicolumn{3}{|l|}{ Motivo para o uso da SNGE } \\
\hline Uso de tubo orotraqueal & 9 & 2,0 \\
\hline Outro & 35 & 7,8 \\
\hline Desnutrição & 41 & 9,2 \\
\hline Inapetência/baixa aceitação via oral & 64 & 14,4 \\
\hline Disfagia & 140 & 31,4 \\
\hline $\begin{array}{l}\text { Queda do sensório/rebaixamento do nível de } \\
\text { consciência/queda do estado geral }\end{array}$ & 157 & 35,2 \\
\hline \multicolumn{3}{|l|}{ Técnicas utilizadas para a introdução da SNE } \\
\hline Endoscopia & 10 & 2,3 \\
\hline Outro & 15 & 3,4 \\
\hline Não se aplicab & 191 & 43,4 \\
\hline Às cegas / à beira leito & 224 & 50,9 \\
\hline
\end{tabular}

Método utilizado para confirmar a posição da extremidade distal da SNE

Somente aspiração do conteúdo gástrico $3 \quad 3 \quad 0,8$

$\begin{array}{lll}\text { Outro } & 9 & 2,4\end{array}$

Ausculta epigástrica e aspiração do conteúdo gástrico $19 \quad 5,0$

Ausculta epigástrica, aspiração do conteúdo $31 \quad 8,2$ gástrico e exame radiológico (raio $X$ )

Ausculta epigástrica e exame radiológico (raio X) $53 \quad 14,1$

Somente ausculta epigástrica $\quad 69 \quad 18,3$

$\begin{array}{lll}\text { Não se aplica }{ }^{b} & 193 & 51,2\end{array}$

${ }^{\mathrm{a} A}$ frequência absoluta não totaliza 447 pacientes devido ao número de dados faltantes. ${ }^{\mathrm{b}} \mathrm{A}$ opção "Não se aplica" foi selecionada quando o paciente foi admitido na enfermaria com a SNE.

Tabela 4 - Análise da associação entre óbito e complexidade assistencial, gravidade, nível de consciência e uso de dispositivo invasivo para respiração (N=447). Ribeirão Preto, 2019.

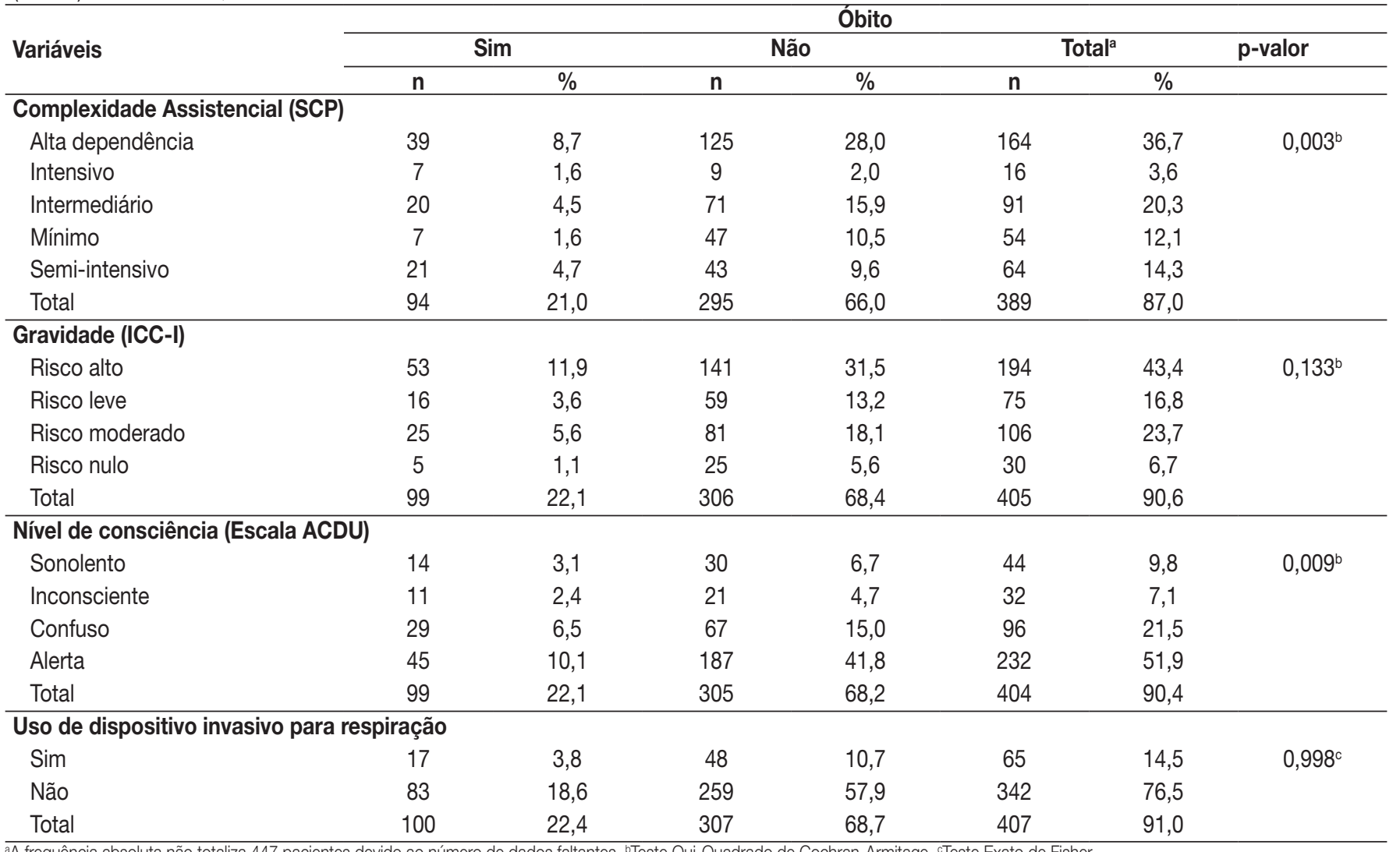

${ }^{\mathrm{a} A}$ frequência absoluta não totaliza 447 pacientes devido ao número de dados faltantes. ${ }^{\mathrm{b}}$ Teste Qui-Quadrado de Cochran-Armitage. ${ }^{\circ}$ Teste Exato de Fisher. 
Sousa FREG et al.

\begin{tabular}{lcccccccc}
\hline \multicolumn{6}{l}{ Tabela 5 - Análise da associação entre óbito e idade, tempo de uso da sonda, tempo de internação e número de comorbidades ( $\mathrm{N}=447)$. Ribeirão Preto, 2019.} \\
\hline & Óbito & Mínimo & Mediana & Média & Máximo & DP & Totala $^{\text {a }}$ & $\boldsymbol{p ~ v a l o r ~}^{\text {b }}$ \\
\hline Idade & Não & 18,75 & 65,76 & 63,64 & 139,75 & 17,47 & 308 & 0,009 \\
& Sim & 25,46 & 70,24 & 68,97 & 104,54 & 14,63 & 100 & \\
\hline Tempo uso sonda & Não & 0,00 & 8,00 & 13,31 & 308,00 & 30,61 & 308 & 0,706 \\
& Sim & 1,00 & 9,00 & 14,16 & 66,00 & 16,04 & 100 & \\
\hline Tempo internação & Não & 1,50 & 11,62 & 16,62 & 372,71 & 24,42 & 308 & 0,699 \\
& Sim & 0,54 & 10,96 & 16,08 & 66,42 & 14,61 & 100 & \\
\hline No comorbidades & Não & 0,00 & 2,00 & 2,22 & 10,00 & 1,76 & 308 & 0,618 \\
& Sim & 0,00 & 2,00 & 2,24 & 11,00 & 2,07 & 100 & \\
\hline
\end{tabular}

DP = Desvio Padrão. ${ }^{a}$ A frequência absoluta não totaliza 447 pacientes devido ao número de dados faltantes. ${ }^{\circledR}$ Teste Qui-Quadrado de Cochran-Armitage.

de internação foi de 16,08 dias (10,96 $\pm 14,61$ dias). Nos pacientes que morreram, a média de comorbidades foi de $2,24(2,00 \pm 2,07)$ (Tabela 5). Quando analisada a associação entre óbito e as variáveis idade, tempo de uso de sonda, tempo de internação e número de comorbidades, verificou-se significância estatística somente para a variável idade $(p=0,009)$ (Tabela 5).

\section{DISCUSSÃO}

A maioria dos pacientes incluídos no estudo era homem, idoso e com ensino fundamental incompleto. Pesquisas prévias apontam para baixa procura de pessoas do sexo masculino pelos serviços de saúde, pois ainda se evidencia a existência de tabus socioculturais que os levam a não reconhecerem suas necessidades de saúde e que encobre a falsa ideia que não estão expostos às doenças. Ademais, o estilo de vida pouco saudável e a subutilização dos serviços por homens refere-se a um problema em muitos países, motivos pelos quais esses fatores de risco os afetam grandemente, pois estão ligados às diferenças de gênero e às normas predominantes de masculinidade ${ }^{16}$.

Neste estudo, 38,4\% dos pacientes com SNE apresentaram ensino fundamental incompleto. A escolaridade também é considerada um fator importante na determinação de saúde das pessoas, visto que, quanto maior o nível de escolaridade, maior a compreensão e tradução dos sinais e sintomas que caracterizam determinadas condições de saúde. $\bigcirc$ nível de instrução demonstra-se como fator importante na execução de programas específicos para a saúde, pois estudo evidenciou que o menor grau de escolaridade dificulta a efetivação de programas preventivos, principalmente aqueles voltados à promoção da saúde e à prevenção de doenças ${ }^{17}$.
A idade média dos pacientes incluídos neste estudo foi de 64 anos. A relação entre idade avançada e uso de SNE foi estabelecida em estudo prévio e se deve, em parte, ao fato de os pacientes apresentarem elevada carga de doença e incapacidade funcional relacionada à demência ${ }^{18}$.

Em relação ao tempo de internação, os pacientes com SNE desse estudo apresentaram média de 17,32 dias, maior que a média encontrada em outro estudo (14,89 dias) em pacientes adultos internados em unidades de clínica médica e com NE' . Este resultado pode ser explicado pela complexidade assistencial e gravidade dos pacientes.

No presente estudo, aproximadamente $42 \%$ dos pacientes foram admitidos nas enfermarias com alterações no nível de consciência e eram altamente dependentes dos cuidados de enfermagem. Ainda, mais de $43 \%$ dos pacientes apresentaram alto risco de óbito e a queda do sensório e disfagia foram os principais motivos para o uso da sonda.

Com o objetivo de caracterizar a população em uso de via alternativa de alimentação, em unidade de emergência do estado do Rio Grande do Sul, estudo apresentou como resultado que as indicações mais prevalentes para NE foram dificuldade de deglutição, redução no nível de consciência, estado geral grave, intubação orotraqueal, recusa ou baixa ingestão oral e/ou inapetência ${ }^{19}$.

Muitas condições médicas agudas e crônicas, como disfagia ou níveis reduzidos de consciência, não permitem o uso de nutrição oral. Nesses casos, a NE deve ser empregada para apoiar as necessidades nutricionais do paciente ${ }^{20}$, sendo importante a avaliação da nutricionista e da fonoaudióloga, que verificam a aceitação, assim como, as condições para deglutir e engolir corretamente, a consistência da dieta e as necessidades energético-proteicas do paciente ${ }^{21}$. 
Destaca-se que, no presente estudo, a maioria dos pacientes foi admitida nos hospitais com doenças do aparelho circulatório (30\%), neoplasias $(14,3 \%)$ e doenças do aparelho respiratório (12,4\%). Ademais, grande parte dos pacientes apresentou doença vascular periférica e hipertensão arterial (34\%), diabetes sem complicação $(18,6 \%)$, doença cerebrovascular $(18,6 \%)$ e doença renal grave ou moderada (1 1,8\%). Esses dados corroboram aqueles encontrados no Sistema de Informação Hospitalar do Sistema Único de Saúde (SUS). No período de janeiro de 2015 a janeiro de 2019, as doenças crônicas não transmissíveis (DCNT) foram as principais causas de morte em adultos, com destaque para as doenças do aparelho respiratório (19\%), doenças do aparelho circulatório (19\%) e neoplasias $(13,3 \%)^{22}$.

Em pesquisa realizada com o objetivo de analisar o perfil de pacientes hospitalizados em uso de NE, identificou-se que $56,3 \%$ apresentaram hipertensão arterial sistêmica (HAS) e 20,1\%, doença neurológica'. Pesquisa prévia também demonstrou associação entre o acidente vascular encefálico e a mortalidade de pacientes em uso de sondas enterais ${ }^{23}$.

Mais de $63 \%$ dos pacientes foram admitidos nas enfermarias de clínica médica com a SNE. Tal resultado evidencia que o uso deste dispositivo é cada vez mais frequente no âmbito domiciliar. Em estudo retrospectivo descritivo realizado em hospital universitário espanhol, verificou que, dentre os 481.000 pacientes admitidos no hospital durante um ano, 141 , sem suporte nutricional prévio, permaneceram em uso de SNE e NE após a alta hospitalar e os cuidados foram mantidos no domicílio ${ }^{24}$.

A taxa de óbito dos pacientes, neste estudo, foi de 22,4\%, inferior aos 33\% encontrada em estudo longitudinal com 30 pacientes em uso de NE em hospital universitário brasileiro ${ }^{25}$. Já outro estudo realizado com a finalidade de traçar o perfil dos pacientes em uso de sonda, internados em um hospital geral de grande porte, a taxa de óbito foi de $26,4 \%$, semeIhante ao do presente estudo'.

Ocorreu maior número de óbitos entre os pacientes altamente dependentes dos cuidados de enfermagem e em risco alto para o óbito. O ICC é considerado um método válido e confiável para medir comorbidades em pesquisas clínicas e é um índice que alerta os profissionais sobre a elevada carga de doenças que os pacientes convivem. Em estudo transversal realizado no estado de Santa Catarina, o número de comorbidades, além do diagnóstico clínico, foi três, sendo HAS, diabetes mellitus e dislipidemias as mais presentes'.

Houve associação entre óbito e complexidade assistencial, visto que a maioria dos pacientes que morreu era altamente dependentes dos cuidados de enfermagem $(8,7 \%)$ ou semi-intensivos $(4,7 \%)$. O SCP proposto por Fugulin ${ }^{13}$ foi desenvolvido para prever o dimensionamento de pessoal necessário para atender às necessidades de assistência de enfermagem. Apesar de não terem sido encontradas na literatura pesquisas que correlacionassem a complexidade assistencial de pacientes em uso de SNE com o óbito, observou-se que, neste estudo, o SCP pode ser utilizado pelos enfermeiros como uma ferramenta gerencial que auxilia na tomada de decisão em relação ao planejamento da assistência prestada aos pacientes em uso de SNE.

Foi identificada associação entre nível de consciência e óbito; neste estudo, $54 \%$ dos pacientes que morreram apresentaram alteração no nível de consciência (confuso, sonolento ou inconsciente). $\bigcirc$ resultado pode ser explicado pela maior gravidade dos pacientes. Alterações do nível de consciência podem sinalizar a deterioração fisiológica em pacientes críticos que necessitam de monitorização especial nas enfermarias ${ }^{26}$.

Houve associação entre óbito e idade, sendo o desfecho mais comum entre os pacientes com média de 68,97 anos. Pesquisas consolidadas comprovaram que o aumento da idade elevou o risco de óbito em pacientes com doenças crônicas. Estudo que visou avaliar os preditores de mortalidade em pacientes com doenças cardíacas apontou que o risco de óbito foi 2,3 vezes maior em pacientes com idade acima de 65 anos $^{27}$. Contudo, em outros estudos, foi verificado que o aumento da idade não alterou a taxa de mortalidade ${ }^{28,29}$. Apesar desta variável ser utilizada em várias ferramentas de estratificação de risco, os resultados apresentados na literatura demonstraram que o impacto da idade na taxa de óbito durante a internação hospitalar ainda é controverso.

\section{Limitações}

Apesar de o estudo ter sido realizado em sete hospitais brasileiros, os resultados podem não representar o perfil da população brasileira internada que faz uso de SNE, tendo em vista as diversidades dos serviços de saúde no país.

\section{CONCLUSÃO}

A maioria dos pacientes em uso de SNE era homem, com média de idade de 64,91 anos, com doença do aparelho circulatório, em estado de alerta, com risco alto para o óbito e altamente dependente dos cuidados de enfermagem. $\bigcirc$ óbito esteve associado à complexidade assistencial, ao nível de consciência e, também, à idade. Faz-se necessária atuação da nutrição e da fonoaudióloga na avaliação destes pacientes.

\section{AGRADECIMENTOS}

Agradecemos a Rhanna Emanuela Fontenele Lima de Carvalho, Thalyła Cardoso Alux Teixeira, Ligia Menezes de Freitas e Janine Koepp, pela assistência na coleta de dados. A investigadora principal também agradece à Sigma Theta Tau International, pelo 2019 Sigma Small Grants. 


\section{REFERÊNCIAS}

1. Ueno E, Koffke M, Voigt VR. Perfil de pacientes hospitalizados em uso de terapia enteral. BRASPEN J. 2018;33(2):194-8.

2. Fan L, Liu Q, Gui L. Efficacy of nonswallow nasogastric tube intubation: a randomised controlled trial. J Clin Nurs. 2016;25(21-22):3326-32.

3. Lamont T, Beaumont C, Fayaz A, Healey F, Huehns T, Law R, et al. Checking placement of nasogastric feeding tubes in adults (interpretation of $\mathrm{x}$ ray images): summary of a safety report from the National Patient Safety Agency. BMJ. 2011;342:d2586.

4. Bortoletto MM, Souza IA, Dias AMN, Almeida NM, Mendonça EG. Perfil sociodemográfico e nutricional de pacientes oncológicos em terapia nutricional enteral. Rev Bras Cancerol. 2018;64(2):141-7.

5. Graciano RDM, Ferretti REL. Nutrição enteral em idosos na Unidade de Terapia Intensiva: prevalência e fatores associados. Geriatria e Gerontol. 2008;2(4):151-5.

6. Cervo AS, Magnago TS, Carollo JB, Chagas BP, Oliveira AS, Urbanetto JS. Adverse events related to the use of enteral nutritional therapy. Rev Gaucha Enferm. 2014;35(2):53-9.

7. Martins AS, Rezende NA, Torres HOG. Sobrevida e complicações em idosos com doenças neurológicas em nutrição enteral. Rev Assoc Med Bras. 2012;58(6):691-7.

8. Word Health Organization (WHO). Global status report on noncommunicable diseases 2014. Geneva: World Health Organization; 2014. p.280.

9. Brasil. Ministério da Saúde, Secretaria de Vigilância em Saúde, Departamento de Análise de Situação de Saúde. Plano de ações estratégicas para o enfrentamento das doenças crônicas não transmissíveis (DCNT) no Brasil 2011-2022. Brasília: Ministério da Saúde; 2011. p.160.

10. Gimenes FRE, Pereira MCA, Prado PRD, Carvalho REFL, Koepp J, Freitas LM, et al. Nasogastric/nasoenteric tube-related incidents in hospitalised patients: a study protocol of a multicentre prospective cohort study. BMJ Open. 2019;9(7):e027967.

11. Favarin SS, Camponogara S. Perfil dos pacientes internados na unidade de terapia intensiva adulto de um hospital universitário. Rev Enferm da UFSM. 2012;2(2):320-9.

12. Charlson ME, Pompei P, Ales KL, MacKenzie CR. Anew method of classifying prognostic comorbidity in longitudinal studies: development and validation. J Chronic Dis. 1987;40(5):373-83.

13. FugulinF.Dimensionamentodepessoaldeenfermagem:avaliação do quadro de pessoal das unidades de internaçãode um hospital de ensino. São Paulo: Escola de Enfermagem da Universidade de São Paulo; 2002.

14. McNarry AF, Goldhill DR. Simple bedside assessment of level of consciousness: comparison of two simple assessment scales with the Glasgow Coma scale. Anaesthesia. 2004;59(1):34-7.

15. Martins M, Blais R, Miranda NN. Avaliação do índice de comorbidade de Charlson em internações da região de Ribeirão Preto, São Paulo, Brasil. Cad Saúde Pública. 2008;24(3):643-52.
16. Barbosa YO, Menezes LPL, Santos JMJ, Cunha JO, Albuquerque TIP, Araújo DC et al. Factors associated with reasons for men not seeking Primary Health Care services. O Mundo da Saúde. 2019;43(3):666-79.

17. Pereira JC, Rocha LTS, Albuquerque SGE, Miranda Neto M, Ribeiro FF. Promoção da saúde do homem: uma experiência exitosa na atenção básica. Rev de APS. 2015;18(1):123-6.

18. Mundi MS, Patel J, McClave SA, Hurt RT. Current perspective for tube feeding in the elderly: from identifying malnutrition to providing of enteral nutrition. Clin Interv Aging. 2018;13:1353-64.

19. Almeida MAF, Mahmud MAI, Finard SA. Profile of patients using nasoenteric probe at the adult emergency service in a public hospital. Clin Biomed Res. 2018;38(3):265-72.

20. Hellerman Itzhaki M, Singer P. Advances in medical nutrition therapy: parenteral nutrition. Nutrients. 2020;12(3):717.

21. Julio R, Mac-Kay APG. Interface entre fonoaudiologia e nutrição: aspectos da alimentação em pacientes idosos hospitalizados. Arq Med Hosp Fac Cienc Med Santa Casa São Paulo. 2005;50(3):110-6.

22. DATASUS. Morbidade hospitalar do SUS - por local de internação - Brasil. Óbitos por sexo segundo região - período: Jan/2018-Fev/2019. Rio de Janeiro: Ministério da Saúde; Secretaria de Gestão Estratégica e Participativa; Departamento de Informática do SUS; Coordenação Geral de Disseminação de Informações em Saúde; 2019.

23. Shin BC, Chun IA, Ryu SY, Oh JE, Choi PK, Kang HG. Association between indication for therapy by nutrition support team and nutritional status. Medicine (Baltimore). 2018;97(52):e13932.

24. Campos-Martín C, García-Torres MD, Castillo-MartínC, Domínguez-Rabadán R, Rabat-Restrepo JM. Patients discharged with home enteral nutrition from a third-level hospital in 2018. Nutrients. 2019;11(11):2570.

25. Gonçalves EC, Morimoto IMI, Ribeiro CSG, Cunha TR, CorradiPerini C. Bioética e direito humano à alimentação adequada na terapia nutricional enteral. Rev Bioet. 2018;26(2):260-70.

26. Westphal GA, Lino AS. Rastreamento sistemático é a base do diagnóstico precoce da sepse grave e choque séptico. Rev Bras Ter Intensiva. 2015;27(2):96-101.

27. Santos CA, Oliveira MAB, Brandi AC, Botelho PHH, Brandi JCM, Santos MA, et al. Fatores de risco para mortalidade de pacientes submetidos à revascularização miocárdica. Braz J Cardiovasc Surg. 2014;29(4):513-20.

28. Aikawa P, Cintra AR, Leite CA, Marques RH, Silva CTM, Afonso MS, et al. Impact of coronary artery bypass grafting in elderly patients. Rev Bras Cir Cardiovasc. 2013;28(1):22-8.

29. Kaufman R, Azevedo VMP, Sá RMG, Geller M, Xavier RMA, Chaves RBM, et al. Epidemiological characteristics and mortality predictors in patients over 70 years submitted to coronary artery bypass grafting. Int $\mathrm{J}$ Cardiovasc Sci. 2018;31(3):258-63.

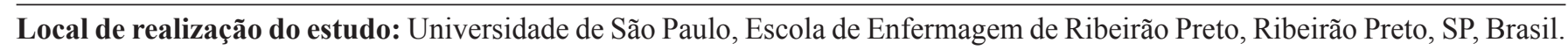

Conflito de interesse: Os autores declaram não haver. 